Berger, Marcus

Kollaboratives Problem-Based Learning. Ein hochschuldidaktischer Ansatz zur Erschließung professionellen Wissens durch problemorientierte und \title{
kollaborative Lernprozesse
}

Tänzer, Sandra [Hrsg.]; Godau, Marc [Hrsg.]; Berger, Marcus [Hrsg.]; Mannhaupt, Gerd [Hrsg.]: Perspektiven auf Hochschullernwerkstätten. Wechselspiele zwischen Individuum, Gemeinschaft, Ding und Raum. Bad Heilbrunn : Verlag Julius Klinkhardt 2019, S. 188-191. - (Lernen und Studieren in Lernwerkstätten)

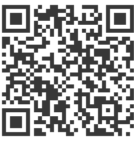

Quellenangabe/ Reference:

Berger, Marcus: Kollaboratives Problem-Based Learning. Ein hochschuldidaktischer Ansatz zur Erschließung professionellen Wissens durch problemorientierte und kollaborative Lernprozesse - In: Tänzer, Sandra [Hrsg.]; Godau, Marc [Hrsg.]; Berger, Marcus [Hrsg.]; Mannhaupt, Gerd [Hrsg.]: Perspektiven auf Hochschullernwerkstätten. Wechselspiele zwischen Individuum, Gemeinschaft, Ding und Raum. Bad Heilbrunn : Verlag Julius Klinkhardt 2019, S. 188-191 - URN: urn:nbn:de:0111-pedocs-201991 - DOI: 10.25656/01:20199

https://nbn-resolving.org/urn:nbn:de:0111-pedocs-201991

https://doi.org/10.25656/01:20199

in Kooperation mit / in cooperation with:

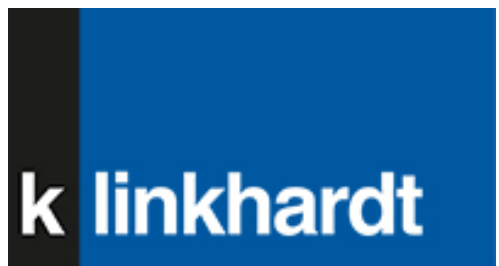

http://www.klinkhardt.de

\section{Nutzungsbedingungen}

Dieses Dokument steht unter folgender Creative Commons-Lizenz: http://creativecommons.org/licenses/by-nc-sa/4.0/deed.de - Sie dürfen das Werk bzw. den Inhalt unter folgenden Bedingungen vervielfältigen, verbreiten und öffentlich zugänglich machen sowie Abwandlungen und Bearbeitungen des Werkes bzw. Inhaltes anfertigen: Sie müssen den Namen des Autors/Rechteinhabers in der von ihm festgelegten Weise nennen. Dieses Werk bzw. der Inhalt darf nicht für kommerzielle Zwecke verwendet werden. Die neu entstandenen Werke bzw. Inhalte dürfen nur unter Verwendung von Lizenzbedingungen weitergegeben werden, die mit denen dieses Lizenzbedingungen weitergegeben werden,

Mit der Verwendung dieses Dokuments erkennen Sie die Nutzungsbedingungen an.

\section{Terms of use}

This document is published under following Creative Commons-License: http://creativecommons.org/licenses/by-nc-sa/4.0/deed.en - You may copy, distribute and transmit, adapt or exhibit the work in the public and alter, transform or change this work as long as you attribute the work in the manner specified by the author or licensor. You are not allowed to make commercial use of the work. If you alter, transform, or change this work in any way, you may distribute the resulting work only under this or a comparable license.

By using this particular document, you accept the above-stated conditions of

\section{Kontakt / Contact:}

\section{DeDOCS}

DIPF | Leibniz-Institut für Bildungsforschung und Bildungsinformation Informationszentrum (IZ) Bildung

E-Mail: pedocs@dipf.de

Internet: www.pedocs.de

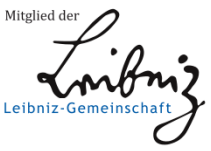




\section{QUALITEACH}

hochSCHULLERNWERKSTATt

\section{Kollaboratives Problem-Based-Learning}

Ein hochschuldidaktischer Ansatz zur Erschließung professionellen Wissens durch problemorientierte und kollaborative Lernprozesse I Marcus Berger

\section{Problemstellung}

- Steigende Komplexität von Wissen erfordert teamorientierte Arbeits- und Lernformen (Honegger \& Notari 2013)

- Nachweise für Effektivität von Gruppenlernprozessen bei Lehrer*innen (Bonsen\& Rolff 2006)

- Kollaborative Lernformen als effektive Variante kollektiven Lernens (Gerstenmayer \& Mandl 2001)

- Nachweise für Förderung von Wissensstrukturen in problemorientierten Lernsettings in Lehramtsausbildung (Stark 2010)

- Notwendigkeit kollektiver Lern- u. Arbeitsformen in der Lehramtsausbildung zur Förderung professionellen Lehrer*innenhandelns (Burow 2000; Bonsen \& Rolff 2006)

- Fehlen von empirisch belegten Anwendungsbeispielen in der Lehramtsausbildung, die problemorientierte Wissenserschließung durch gezielte kollaborative Herangehensweisen fördern.

\section{Fragestellungen}

Wie muss eine geeignete hochschuldidaktische Lernumgebung gestaltet sein, die das kollektive Arbeiten im Team fördert und auf diese Weise gezielt Aspekte professionellen Wissens angehender Lehrer*innen steigert?

Erfolgt in diesem Setting die Transformation von verteilten in geteilte Wissensstrukturen?

\section{Ausgangsmodelle}

Problem Based Learning 7 Sprung

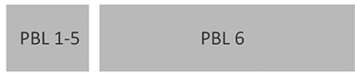

PBL 7

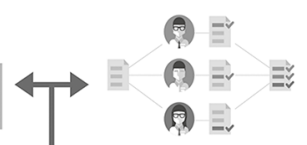

Analyse

Wissensaneignung

vert. Probl.anal.

Kooperative Zusammenarbeit

vgl. Weber 200

\section{Forschungsansatz}

Design-Based-Research

(Reinmann 2016; Euler 2014)

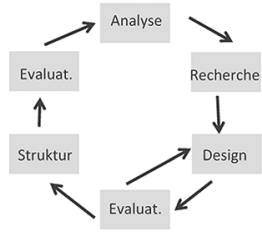

summativ:

Weitere quasiexperimentelle Studie im Versuchs- Kontroll- gruppendesign(Performanz-test: deklaratives + prozedurales Wissen im WS

$18 / 19$ und SoSe $19 \mathrm{~N}=$ ca.300)

formativ:

Prozessbegleitende Untersuchung einer Arbeitsgruppe um kollaborative Wissenstransformation zu beschreiben

QUALITEACH wird im Rahmen der
gemeinsamen "Qualitătsoffensive
Lehrerbildung" von Bund und Ländern
aus Mitteln des Bundesministeriums fürutive
Bildung und Forschung gefördert."

Abb. 1: Posterbeitrag Kollaboratives Problem-Based Learning leitfadengestütze Gruppeninterviews in Seminaren der Deutschdidaktik und Sachunterrichtsdidaktik im SoSe 2017, WS 2017/18, SoSe 2018, WS 2018/19

\section{Ausblick}

5. aktueller Stand

Kollaboratives PBL

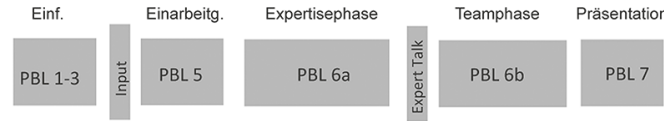

\section{7}




\section{Kollaboratives Problem-Based Learning. Ein hochschuldidaktischer Ansatz zur Erschließung professionellen Wissens durch problemorientierte und kollaborative Lernprozesse}

\section{Bedarf kollaborativer Lernprozesse zur Wissenserschließung}

Die stets steigende Komplexität von Wissen fordert unter anderem ein teamorientiertes und multiperspektivisches Arbeiten (Honegger \& Notari 2013, 31). Aus hochschuldidaktischer Perspektive besteht eine Notwendigkeit, dass Lehrer*innen bereits in ihrer Ausbildung befähigt werden sollten, kollektive Lernformen zu nutzen (Burow 2000, 31-34), da diese als Aspekt professionellen Lehrer*innenhandelns verstanden werden können und diesbezüglich Effekte nachweisbar sind (ebd.; Bonsen \& Rolff 2006, 168). Empirische Forschungen zum situierten Lernen heben dabei die Bedeutung des kollaborativen Lernens im Bildungskontext hervor und betonen die Verzahnung individuell distribuierten und sozial geteilten Wissens (Gerstenmayer \& Mandl 2001, 14, 15). Geteiltes Wissen im Sinne eines gemeinsamen Verfügens gilt als wichtiger Faktor für kollektive Problemlöseprozesse (Thalemann 2003, 2).

Um Wissenserwerb und kollaborative Lernprozesse bezüglich des situierten Lernens hochschuldidaktisch einzubetten, bieten sich problemorientierte Lernsettings wie das Konzept des Problem-Based Learnings an (Weber 2007, 17,189). Sie ermöglichen strukturell ein gruppenbasiertes Arbeiten (ebd.,11) und bieten gleichzeitig Belege für eine effektive Förderung u.a. von Wissensstrukturen in der Lehramtsausbildung (Stark 2010, 558-560).

Bisher fehlt es allerdings an konkreten und empirisch belegten, hochschuldidaktischen Anwendungsbeispielen im Bereich der Lehrer*innenausbildung, die problemorientierte Wissenserschließung durch gezielte kollaborative Herangehensweisen fördern.

Für das hier dargestellte Forschungsprojekt ergeben sich die Fragen, (1) wie eine geeignete hochschuldidaktische Lernumgebung gestaltet sein muss, die das kollaborative Arbeiten im Team explizit fördert und auf diese Weise gezielt Aspekte professionellen Wissens angehender Lehrer*innen steigert und (2) ob in einem 
solchen Setting die Transformation von verteilten in geteilte Wissensstrukturen durch kollaborative Arbeitsformen erfolgt.

\section{Forschungsansatz}

Um einen theoretischen Erkenntnisgewinn mit einem bildungspraktischen Nutzen zu verknüpfen, erscheint ein Forschungsansatz sinnvoll, der sich über die klassische Trennung von Anwendungs- und Grundlagenforschung hinwegsetzt. Design-Based-Research (DBR) ermöglicht es, die Entwicklung einer Intervention (Design) als fortwährenden Bestandteil des Forschungsprozesses zu betrachten. Erste Ergebnisse werden formativ evaluiert, ggf. überarbeitet (Re-Design) und die ausgereifte Intervention summativ evaluiert (Reinmann 2016, 2-4). Auf diese Weise können nicht nur Probleme aus der Bildungspraxis gelöst, sondern gleichzeitig tragfähige Theorien entwickelt werden, die wissenschaftliche Erkenntnisse zum Lehren und Lernen fördern (Reinmann 2005, 62).

\section{Design der Intervention}

Als Adaptionsgrundlage dient das Modell des Problem-Based Learnings in der Variante des Siebensprungs (Weber 2007, 29-40). Bereits in seiner ursprünglichen Konzeption sind gruppenbasierte Lernprozesse fest verankert (ebd., 30). Ferner ist es möglich, das Modell dahingehend abzuändern, dass eine gezielte strukturelle Unterstützung kooperativer und kollaborativer Lernprozesse ermöglicht wird. Kooperative Zusammenarbeit meint in diesem Zusammenhang eine starke Funktions- und Arbeitsteilung mit additivem Charakter. Kollaborative Zusammenarbeit wird hingegen als ein synchroner Prozess der ko-konstruktiven Wissensgenerierung betrachtet. Es werden nonsummative Prozesse befördert, die im Ergebnis mehr beinhalten können als die reine Addition von Einzelleistungen (Bornemann 2012, 77-79).

Die konkrete didaktische Umsetzung wird seit dem Sommersemester 2017 regelmäßig in Lehrveranstaltungen der Grundschuldidaktik Deutsch im Bereich Schriftspracherwerb der Universität Erfurt weiterentwickelt, erprobt und evaluiert. 


\section{Evaluation}

Das Modell befindet sich derzeit im Re-Design. Mit Bezug auf leitfadengestützte Gruppeninterviews wird es weiter adaptiert und angepasst.

Im Versuchs- Kontrollgruppendesign wurden bereits in Pilotstudien psychometrische Parameter zur Erfassung pädagogischer Professionalität (Baumert \& Kunter 2011) erhoben. Im Bereich der Erschließung prozeduralen Wissens (eigene Testkonstruktion) konnten bereits erste positive Ergebnisse erzielt werden.

Das Design der Intervention wird im Wintersemester 2018/19 abgeschlossen. In einer weiteren quasiexperimentellen Studie sollen im Wintersemester 2018/19 und im Sommersemester 2019 weitere summative Erhebungen stattfinden $(\mathrm{N}=300)$.

\section{Literatur}

Baumert, Jürgen \& Kunter, Mareike (2011): Professionelle Kompetenz von Lehrkräften. Ergebnisse des Forschungsprogramms COACTIV. Münster: Waxmann.

Bonsen, Martin \& Rolff, Hans Günter. (2006): Professionelle Lerngemeinschaften von Lehrerinnen und Lehrern. In: Zeitschrift für Pädagogik, 52 Jg., H. 2., Frankfurt a. M.: Beltz, 167-184.

Bornemann, Stefan (2012): Kooperation und Kollaboration. Das kreative Feld als Weg zu innovativer Teamarbeit. Wiesbaden: Springer VS.

Burow, Olaf Axel (2000): Kreativität im Team. Ein neues Lehrerbild für die Wissensgesellschaft. In: Pädagogik. H. 6, 31-34.

Gerstenmaier, Jochen \& Mandl, Heinz (2001): Methodologie und Empirie zum situierten Lernen (Forschungsbericht 137). München: Ludwig Maximilian Universität, Lehrstuhl für empirische Pädagogik und Pädagogische Psychologie. Methoden, Befunde und Perspektiven. Münster: Waxmann, 35-61.

Honegger, Beat D. \& Notari, Michele (2013): Das Wiki-Prinzip. In: Notari, Michele \& Honegger, Beat D. (Hrsg.): Der WIKI Weg des Lernens. Gestalten und Begleiten von Lernprozessen mit digitalen Kollaborationswerkzeugen. Bern: hep -verlag, 20-35.

Reinmann, Gabi (2016): Design-Based-Research am Beispiel hochschuldidaktischer Forschung. Redemanuskript vom 18.11.2016. https://gabi-reinmann.de/wp-content/uploads/2016/11/Vortrag Berlin_Nov2016.pdf [letzter Zugriff am 11.09.2018].

Reinmann, Gabi (2005): Innovation ohne Forschung. Ein Plädoyer für den Design-Based-Research Ansatz in der Lehr-Lernforschung. In: Unterrichtswissenschaft, 33 Jg., H. 1, 52-69.

Stark, Robin; Herzmann, Petra \& Krause, Ulrike-Marie (2010): Effekte integrierter Lernumgebungen - Vergleich problembasierter und instruktonsorientierter Seminarkonzeptionenin der Lehrerbildung. In: Zeitschrift für Pädagogik, 56 Jg. H. 4, Frankfurt Main: Beltz, 548-563.

Thalemann, Susanne (2003): Die Rolle geteilten Wissens beim netzbasierten kollaborativen Problemlösen. Albert-Ludwigs-Universität Freiburg i. Brsg., Wirtschafts- und Verhaltenswissenschaftliche Fakultät. https://freidok.uni-freiburg.de/dnb/download/1327 [letzter Zugriff am 11.09.2018].

Weber, Agnes (2007): Problem-Based Learning. Ein Handbuch für Ausbildung auf der Sekundarstufe 2 und der Tertiärstufe. Bern: hep verlag. 